\title{
GRAINE balloon-borne experiment in 2015 : Observations with a high angular resolution gamma-ray telescope
}

\author{
Hiroki Rokujo* \\ Nagoya University \\ E-mail: rokujoeflab.phys.naqoya-u.ac.jp
}

GRAINE collaboration

Aichi University of Education, ISAS/JAXA, Utsunomiya University, Okayama University of

Science, Kobe University, and Nagoya University

\begin{abstract}
Observations of cosmic gamma rays are important to promote an understanding of such highenergy objects and phenomena in the universe. Since 2008, the Large Area Telescope on the Fermi satellite has surveyed the whole gamma-ray sky in the sub-GeV/GeV energy region, and accumulated a large amount of data. However, observations at low galactic latitudes remain difficult because of a lack of angular resolution, an increase of background flux originating from galactic diffuse gamma rays, etc. The Gamma-Ray Astro-Imager with Nuclear Emulsion (GRAINE) is a gamma-ray observation project with a new balloon-borne emulsion gamma-ray telescope. Nuclear emulsion is a high-resolution 3D tracking device. It determines the incident angle with $0.1^{\circ}$ resolution for $1 \mathrm{GeV}$ gamma rays $\left(1.0^{\circ}\right.$ for $\left.100 \mathrm{MeV}\right)$, and has polarization sensitivity. The goal of the GRAINE is to achieve precise observations of gamma-ray sources, especially in the galactic plane, by repeating long-duration balloon flights with large-aperture-area $\left(10 \mathrm{~m}^{2}\right)$ high-resolution emulsion telescopes. In May 2015, we performed a balloon-borne experiment in Alice Springs, Australia, in order to demonstrate the imaging performance of our telescope. The emulsion telescope that has an aperture area of $0.4 \mathrm{~m}^{2}$ was employed in this experiment. It observed the Vela pulsar (the brightest gamma-ray source in the GeV sky) at an altitude of $37 \mathrm{~km}$ for 6 hours out of the flight duration of 14 hours. In this paper, we report the data analysis, especially evaluations of the detector performance in the GRAINE 2015 experiment by observing an external calibration source.
\end{abstract}

35th International Cosmic Ray Conference

12-20 July, 2017

Bexco, Busan, Korea

${ }^{*}$ Speaker. 


\section{Introduction}

Observations of cosmic gamma rays emitted from black holes, pulsars, supernova remnants, etc. are important to promote an understanding of such high-energy objects and phenomena in the universe. AGILE [ $\mathrm{W}$ ] launched in 2007 and the Large-Area Telescope on the Fermi Gamma-ray Space Telescope (Fermi-LAT) [[] launched in 2008 have surveyed the sub-GeV/GeV gamma-ray sky. They have achieved good results and contributed to the development of gamma-ray astronomy by detection of more than 3000 gamma-ray sources [B] and discovery of cosmic-ray proton acceleration in supernova remnants [ [ 9 , 国].

On the other hand, new issues have come to light. Observations at low galactic latitudes remain difficult because of a lack of angular resolution, an increase of background flux originating from galactic diffuse gamma rays, etc. A large percentage of the gamma-ray sources detected in the galactic plane are still classified as unassociated sources [3]. Furthermore, an unexpected gammaray excess in the galactic center region has been reported [目].

The goal of the Gamma-Ray Astro-Imager with Nuclear Emulsion (GRAINE) project is to achieve precise observations of gamma-ray sources by a balloon-borne gamma-ray telescope. The high-angular-resolution gamma-ray telescope, called an emulsion telescope, consists of nuclear emulsion films. Because nuclear emulsions can determine the angles of electrons and positrons at the beginning point of a pair-creation interaction $\left(\gamma \rightarrow \mathrm{e}^{+}+\mathrm{e}^{-}\right)$, the angular resolution for gamma rays $(10 \mathrm{MeV}-10 \mathrm{GeV})$ is about one order of magnitude higher than that of Fermi-LAT $\left(0.9^{\circ}\right.$ at 100 $\mathrm{MeV}, 0.1^{\circ}$ at $\left.1 \mathrm{GeV}\right)$.

Our second balloon experiment, GRAINE 2015, was performed on May 12, 2015. Its goal was to detect gamma-ray sources, and demonstrate the imaging performance of the emulsion telescope in the $100 \mathrm{MeV}$ energy region. The balloon was launched from the Alice Springs balloon-launching station, Australia at 6:33 Australian Central Standard Time (ACST), and reached $37.2 \mathrm{~km}$ altitude at 8:50. At 14:15 the Vela pulsar entered the field of view of the emulsion telescope. The telescope observed the target for about $6 \mathrm{~h}$, then the balloon released the gondola at 20:22. The total flight duration in this experiment was $14.4 \mathrm{~h}$, with $11.5 \mathrm{~h}$ of level flight at 36.0-37.4 km altitude and 4. $-3.8 \mathrm{hPa}$ residual atmospheric pressure. More detailed information is presented in [प].

In this paper, we report the data analysis, especially evaluations of the detector performance in the GRAINE 2015 experiment by observing an external calibration source.

\section{The detector in the GRAINE-2015 balloon experiment}

Figure $\square$ shows the photographs and a cross-sectional view of the emulsion gamma-ray telescope employed in GRAINE 2015. We enlarged the aperture area of the second telescope to 3780 $\mathrm{cm}^{2}$, which is 29 times larger than that of the first telescope. The converter consists of 100 highsensitivity emulsion films. The thicknesses of the plastic layer and the both-side emulsion layers in a film are $180 \mu \mathrm{m}$ and $70 \mu \mathrm{m}$, respectively. The total thickness and the radiation length are 32 $\mathrm{mm}$ and $0.53 X_{0}$, respectively. Thirty-four percent of vertically incident gamma rays convert to electron-positron pairs, and these tracks are recorded in the emulsion films. The area of a converter film is $37.8 \mathrm{~cm} \times 25 \mathrm{~cm}$. Four units with the same structure were employed. An alignment unit (two or three emulsion films vacuum-packed with an aluminum honeycomb panel) was placed on 


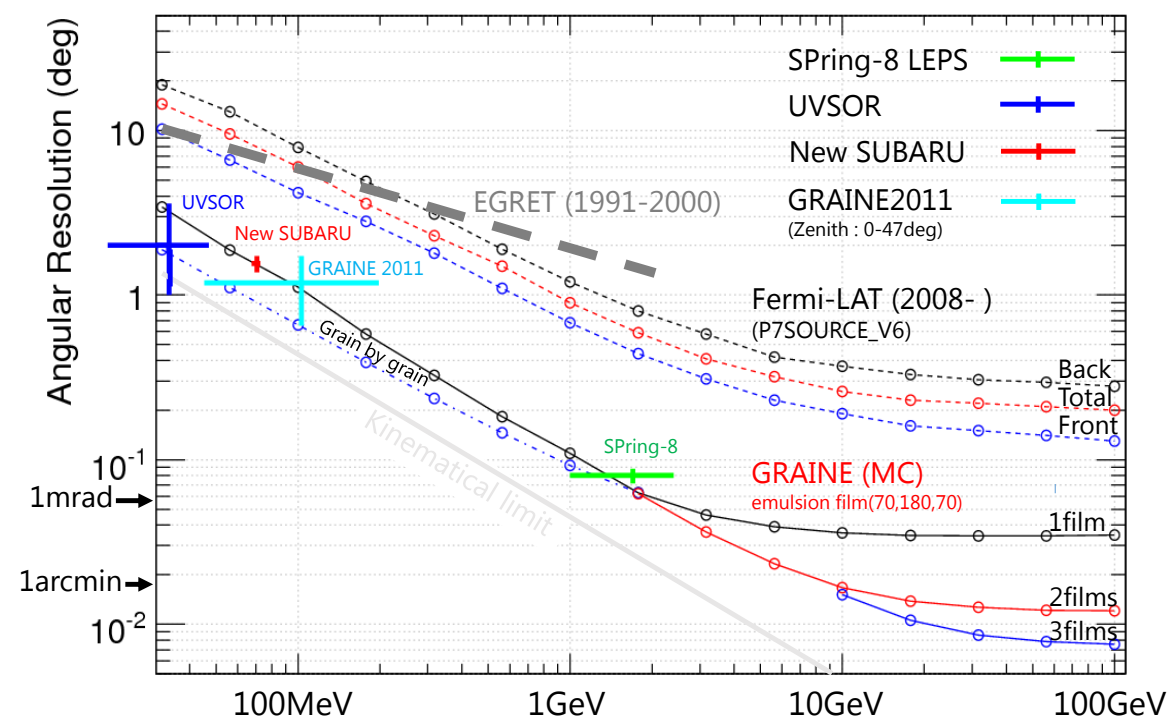

Figure 1: Angular resolution of the emulsion gamma-ray telescope (the lines show simulation results, and the data points with error bars show experimental results) for Fermi-LAT (dotted lines), and EGRET (a broken line).

the top of each converter unit. The alignment unit is the standard surface of the detector system, and the angle of each track recorded in the converter is calibrated by high momentum tracks penetrating both the alignment unit and the converter. A multi-stage shifter system that functions as a time stamper [ [8] was placed at the bottom of the converter. Between two and four emulsion films were mounted on each movable stage. Three stages are driven by stepping motors; throughout the duration of the observation, they slide cyclically like an analog clock, and create independent combinations of the stage position. The energy measurement for multi-GeV gamma-ray events was performed by an analysis of the calorimeter, which has a sandwich structure consisting of sixteen emulsion films and fifteen 1-mm-thick stainless steel plates. The thickness and radiation length were $19.3 \mathrm{~mm}$ and $0.90 X_{0}$, respectively. The balloon also carried three star trackers as attitude monitors, a balloon-style pressure vessel to maintain the vacuum-packed emulsion chamber, and several sensors (for GPS, temperature, pressure, etc.) as its payload.

\section{Demonstration of gamma-ray imaging with emulsion telescope}

\section{1 gamma-ray event selection}

The acquisition of data from the emulsion films was performed by the latest scanning system, Hyper Track Selector (HTS), which was developed at Nagoya University [Q]. The first practical data acquisition using HTS went smoothly, and it took about three months to finish $41 \mathrm{~m}^{2}$ of the converter films and the time-stamper films. The analysis of gamma-ray event $\left(\gamma \rightarrow e^{+}+e^{-}\right)$ selection is described in [प]]. The density of the number of tracks read out by HTS was 400 tracks $/ \mathrm{mm}^{2}$ in each film. After event selection, the density decreased by a factor of $\sim 2000$, down to 0.2 tracks $/ \mathrm{mm}^{2}$. In the current conventional selection, the event-pickup efficiency in the converter was estimated at $65 \%$ and $83 \%$ for $100 \mathrm{MeV}$ and $200 \mathrm{MeV}$ gamma rays, respectively. So far, $75 \%$ 

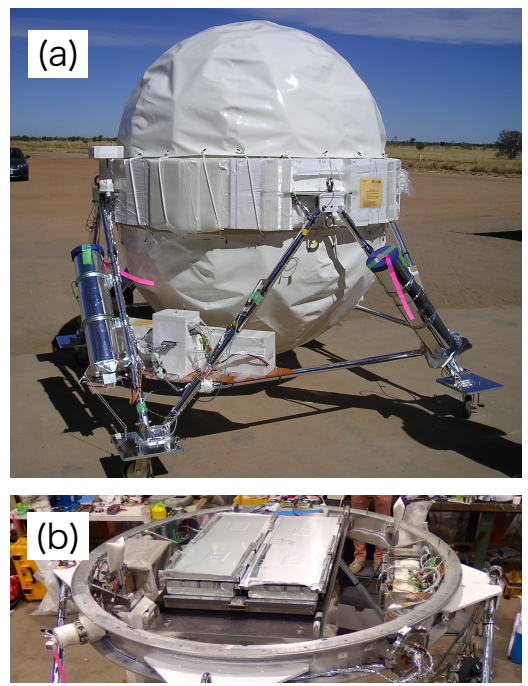
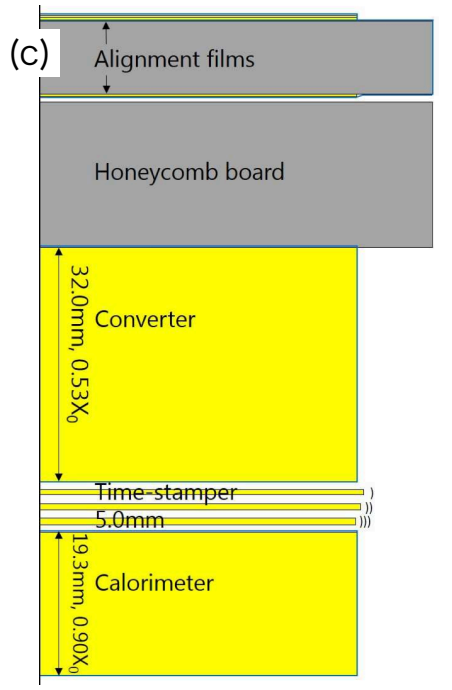

Figure 2: (a) Photograph of the gondola. A spherical container with an inside diameter of $1.6 \mathrm{~m}$ functions as a balloonstyle pressure vessel. Three star cameras as attitude monitors were mounted on the trusses (one of them is on the far side in this picture). (b) Inside view of the pressure vessel. The emulsion gamma-ray telescope was placed at the center of a ring. The total aperture area of four units was 3780 $\mathrm{cm}^{2}$. (c) Cross sectional view of the emulsion gamma-ray telescope, which consists of alignment films, the converter, the time stamper, and the calorimeter.

of the data processing (data acquisition by the scanning system, and gamma-ray event detection in the offline process) has been completed.

\subsection{External gamma-ray source for imaging demonstration}

Figure [3] shows photographs of the gondola suspended by a crane truck, and of an aluminum plate, called a launching plate, that connected the payload to the huge balloon. The crane held the launching plate and released it at the time of launching. After launching, the plate was situated $4.4 \mathrm{~m}$ above the emulsion telescope. The plate was exposed to cosmic rays, mainly protons, at the observation altitudes. When cosmic rays interact with the materials, secondary particles including gamma rays are created. Thus, the launching plate became an external gamma-ray source for calibration of the emulsion telescope during the flight.

Figure [ 3 shows pictures of the gondola hanged by a crane truck and an aluminum plate, called a launching plate, that connected the payload to the huge balloon. The crane held the launching plate and released it at the timing of launching. After launching, the plate existed $4.4 \mathrm{~m}$ above the emulsion telescope. Cosmic rays, mainly protons, were exposed to the plate at the observation altitudes. When cosmic rays interact with the materials, secondary particles including gamma rays are created. Thus, the launching plate became an external gamma-ray source for calibration of the emulsion telescope in the flight.

\subsection{Result of gamma-ray imaging with the emulsion telescope}

The gamma-ray image of the launching plate is not created by the angular distribution of detected gamma-ray events, because the aperture size of the emulsion telescope is not negligible, compared with the distance to the launching plate. Therefore, we mapped the extrapolated positions, which are cross points of gamma-ray vectors and a plane at a height of the launching plate 


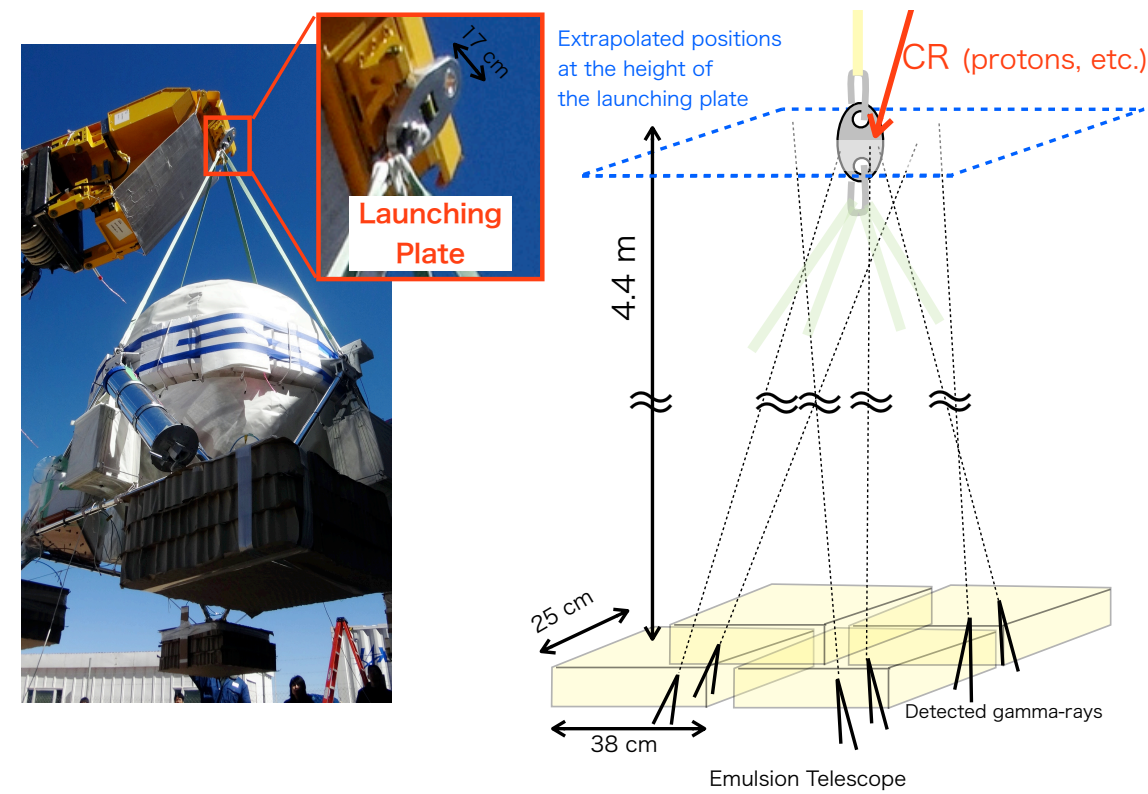

Figure 3: The photographs on the left show the gondola of GRAINE 2015 and the launching plate. The schematic view on the right shows the positional relationship between the emulsion telescope and the launching plate. The square drawn with blue dotted lines indicates a plane at the height of the launching plate.

(see the right schematic view in Figure (3). The extrapolated positions $X_{\text {ext. }}$ and $Y_{\text {ext. }}$ are determined by the following equations:

$$
\begin{gathered}
X_{\text {ext. }}=\tan \theta_{X} \times\left(Z_{c n v .}+L\right)+X_{c n v .} \\
Y_{\text {ext. }}=\tan \theta_{Y} \times\left(Z_{c n v .}+L\right)+Y_{c n v .}
\end{gathered}
$$

where a three-dimensional position $\left(X_{c n v}, Y_{c n v}, Z_{c n v}\right)$ corresponds to a conversion point in the detector coordinates, $\theta_{X}$ and $\theta_{Y}$ are projection angles of the gamma ray (the origin of angles is defined as the zenith), and $L$ is the height of the launching plate from the surface of the detector. Figure $\$$ shows the distributions of extrapolated positions. The energy range is set at 100-300 MeV. The left image is the result of counting the number of events with running bins (the region in each bin is a circle with a radius of $5 \mathrm{~cm}$ ). The right figure is a distribution of $R^{2}$, where $R$ is the distance in the extrapolated position space between each gamma-ray event and the center of the detected image. The background distribution, which can be estimated from counting at off-source regions, is subtracted from the entries in each bin. A clear excess in the number of events was observed and we succeeded in imaging the launching plate by gamma rays.

\subsection{Discussion}

\subsubsection{Monte Carlo simulation}

To reproduce gamma rays emitted from the region of the launching plate we performed the following Monte Carlo (MC) simulation. Targets that imitated the shapes, materials and masses of the launching plate (4.6 kg, aluminum) and shackles $(3.1 \times 2 \mathrm{~kg}$, stainless steel) were created and placed $4.4 \mathrm{~m}$ above the detector in in the Geant 4.10 .01 simulation software package. Incident particles were generated at a plane over the targets. Information concerning gamma rays that 

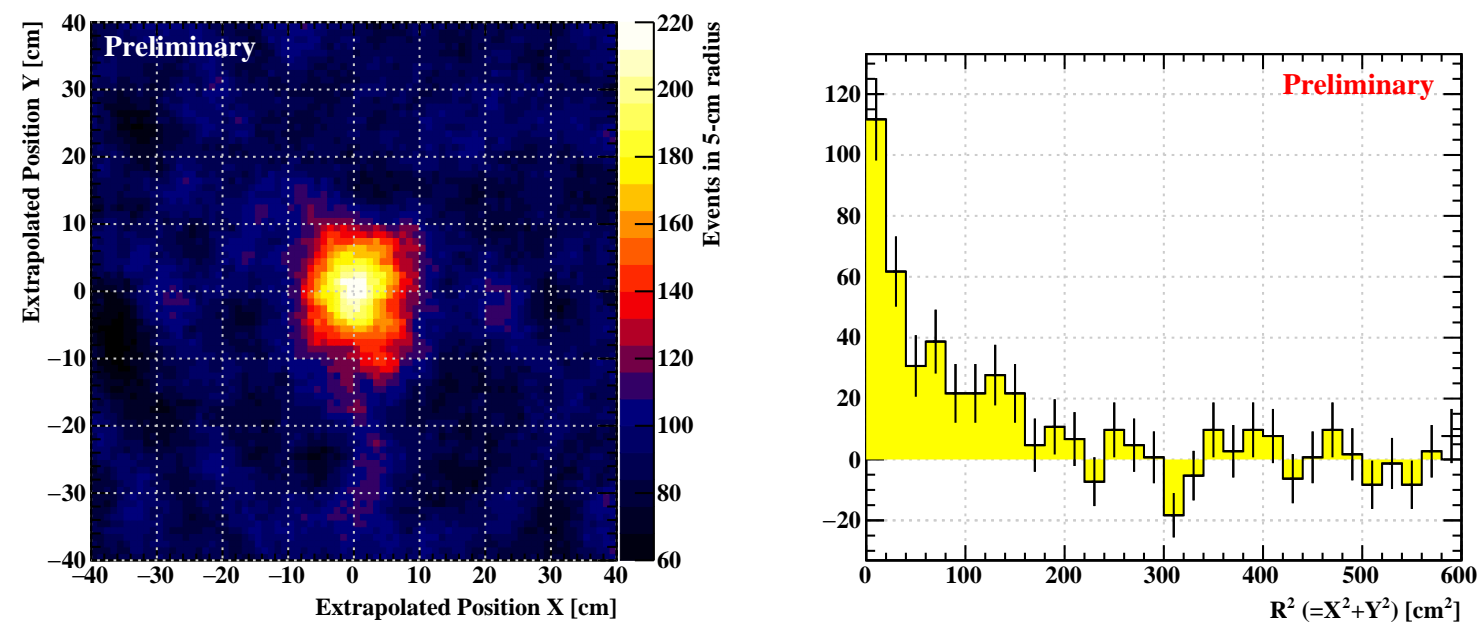

Figure 4: The left image shows an extrapolated position distribution as a result of counting the number of events with running bins (5-cm-radius circles). The energy range is set at 100-300 MeV. The figure on the right is the distribution of $R^{2}$ after subtraction of the background distribution estimated from counting in off-source regions.

were produced by the interactions was output if the gamma rays reached all the way down to the detector surface. The QGSP_BERT package was used for PhysicsList. We adopted results of calculation by the HKKM model [प] for the estimation of energy spectra, zenith angle distributions, and azimuthal angle distributions of cosmic rays at the observation altitudes in Australia, and applied them to incident particles (protons, alphas, electrons, positrons, and gamma rays) in the MC simulation.

\subsubsection{Flux measurement}

We defined $F_{\text {plate }}$ as the flux of gamma rays from the launching plate. Signal events in the on-source region $(R<20 \mathrm{~cm})$ were calculated by subtracting the average number in the off-source regions. Figure $\square$ shows the results of measuring $F_{\text {plate }}$ (red plot) and the simulated values (blue plot). The emulsion telescope employed in GRAINE 2015 observed gamma rays from the launching plate with the expected flux.

\subsubsection{Imaging resolution}

Figure 6 shows the $\mathrm{R}^{2}$ distributions estimated by the $\mathrm{MC}$ simulation events and the distribution of the observation data. The gray plot indicates the expanse of a gamma-ray image of the plate if MC-true angles of detected gamma-ray events are used for the calculation of the extrapolated positions (Equations $[$.$] and [.2$ ). The red, blue, and green plots show the results of smearing the gamma-ray angles with angular resolutions that are 1.0, 1.5, and 2.0 times as much, respectively, as the expected performance in Figure $\mathbb{W}$. Here, a simple 2-D Gaussian was used for the point spread function. It is difficult to discuss the exact angular resolution of the emulsion telescope because the launching plate is not a point source. However, the red or blue plots reproduce the observed data well. Therefore, we conclude that the emulsion telescope had an angular resolution in the 100 $\mathrm{MeV}$ region that met our expectations. 


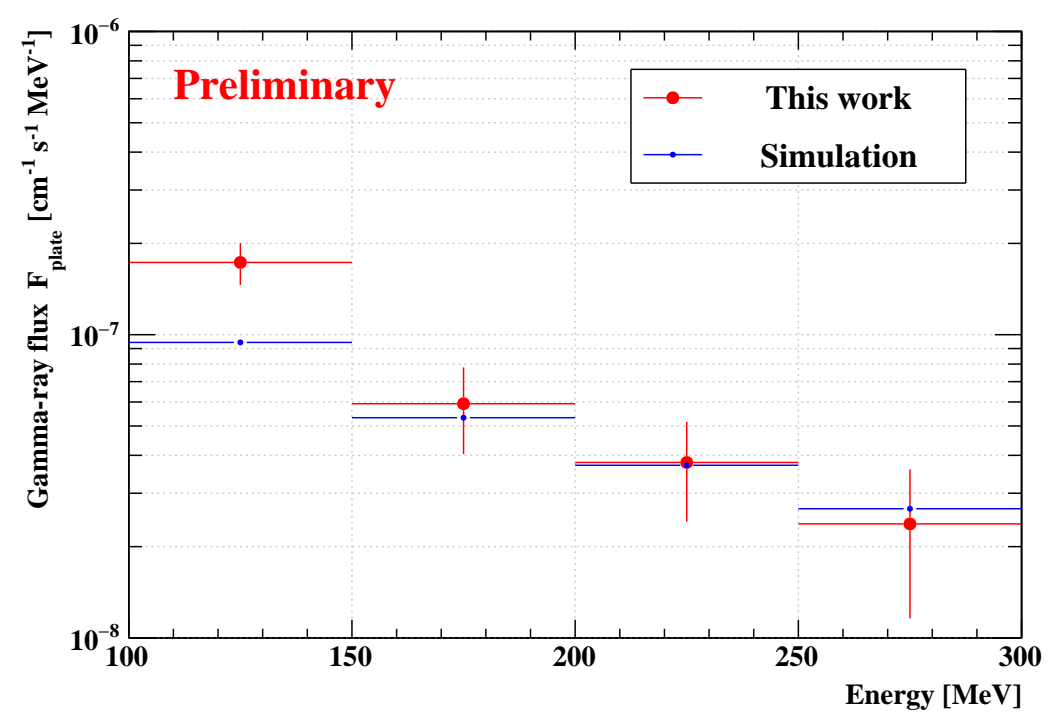

Figure 5: Flux of gamma rays from the launching plate region at the position of the emulsion telescope.

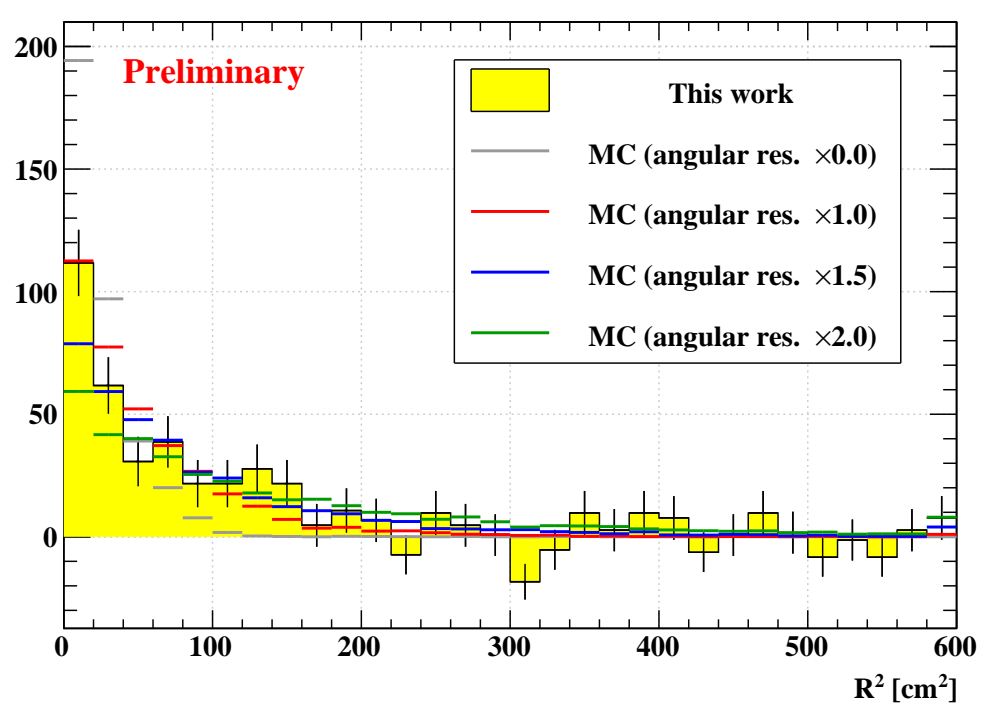

Figure 6: $R^{2}$ distributions estimated by MC simulation events and the distribution of the observation data.

\section{Summary}

GRAINE is a precise gamma-ray observation project using a balloon-borne emulsion telescope. Advantages of the telescope are higher angular resolution, polarization sensitivity, and a large-aperture area. The second balloon experiment was performed in 2015, the flight data analysis is ongoing. We evaluated the detector performance by observing an external gamma-ray source: the launching plate. A clear excess in the number of events was detected at the region of the plate in the extrapolated position space, and we succeeded in imaging by gamma rays. The flux from the 
source region and the expanse of the image were consistent with predictions of the MC simulation. We demonstrated that the emulsion telescope had the expected performance in the $100 \mathrm{MeV}$ region during the balloon flight in GRAINE 2015.

We are preparing for the next balloon experiment planned for April 2018 [ㄴ2]. The goal of that experiment will be to detect celestial gamma-ray sources. After the final confirmation of the overall performance of the balloon-borne emulsion telescope, the GRAINE project will enter the scientific observation phase in 2021.

\section{Acknowledgments}

The scientific balloon (DAIKIKYU) flight opportunity was provided by ISAS, JAXA. We would like to thank Dr. M. Honda (ICRR, University of Tokyo) for calculation of cosmic-ray flux at the balloon altitudes. This work was supported by JSPS. KAKENHI (Grant Numbers 20244031, 26105510, 26247039, 26800138, and 16K17691) and a Grant-in-Aid for JSPS. Fellows.

\section{References}

[1] M. Tavani et al., Astron. Astrophys. 502, 995 (2009).

[2] W. B. Atwood et al., Astrophys. J. 697, 1071 (2009).

[3] F. Acero, et al., The Astrophysical Journal Supplement Series 218(2), 23, (2015).

[4] A. Giuliani, et al., The Astrophysical Journal Letters 742(2), L30, (2011).

[5] M. Ackermann, et al., Science 339(6121), 807-811, (2013).

[6] T. Daylan, et al. Physics of the Dark Universe 12 ,1-23, (2016).

[7] S. Takahashi et al., Prog. Theor. Exp. Phys. 2016, 073 F01 (2016).

[8] S. Takahashi et al., Nucl. Instrum. Meth. A 620, 192 (2010).

[9] M. Yoshiomto et al., arXiv:1704.06814 [physics.ins-det].

[10] H. Rokujo, et al., EPJ Web of Conferences 145, 06002 (2017).

[11] http://www.icrr.u-tokyo.ac.jp/ rmhonda/

[12] S. Takahashi, et al., Proceedings of ICRC 2017, PoS(ICRC2017)835 